Original article

\title{
The impact of transesophageal echocardiography based protocol for management of adults in the sitting position on the incidence of clinically significant venous air embolism
}

\author{
Dmitry A. Averyanov, Roman S. Lakotko, Aleksey V. Shchyogolev, Dmitry V. Svistov, Aleksey I. Gayvoronsky
}

S.M. Kirov Military Medical Academy, Saint-Petersburg, Russia

Received 25 February 2019, Revised 4 November 2019, Accepted 13 January 2020

(C) 2019, Averyanov D.A., Lakotko R.S., Shchyogolev A.V., Svistov D.V., Gayvoronsky A.I.

(C) 2019, Russian Open Medical Journal

\begin{abstract}
The purpose of the study was to evaluate the impact of transesophageal echocardiography (TEE) - based protocol for management of adults in the sitting position during elective neurosurgical interventions on the incidence of clinically significant venous air embolism (VAE).

Material and Methods - The study involved 155 adult neurosurgery patients (70 in prospective group and 85 in retrospective group). Surgery in both groups was done in the sitting position. In the prospective group TEE-based protocol was used. Retrospective group served as control. The primary endpoint was considered to be a decrease in the frequency of clinically significant VAE in the prospective group in comparison with the retrospective one. In the prospective group, VAE with Tuebingen grade 3-5 was considered clinically significant. The PFO incidence and severity and the effect of the number of episodes of VAE per case on its maximum severity during surgery were also analyzed. Any complication in the postoperative period believed to be associated with the position of the patient on the table during the surgery was recorded.

Results - The incidence of the clinically significant VAE in the retrospective group was $23.5 \%(95 \% \mathrm{Cl} 15-34)$ and was $16.4 \%$ higher than the frequency in the prospective group (chi-square $=7.6197, \mathrm{df}=1, \mathrm{p}=0.005) .50 \%(95 \% \mathrm{Cl} 38-62$ ) of patients in prospective group developed $\mathrm{VAE}$ during surgery. In 16 cases, the number of episodes was more than one. The number of episodes of VAE in the observation was reliably associated with the maximum severity of VAE during the observation $(Z=4.11 ; p<0.001)$. A moderate strength relationship was determined between them (SomersDelta $=0.43 ; 95 \% \mathrm{Cl} 0.17-0.7$ ). Not a single case of paradoxical air embolism was detected in a series of observations. None of the patients has got a neurological deficit or cardiopulmonary complications associated with the position on the surgical table in the postoperative period. Pneumocephalus was found in $100 \%$ of cases on head computed tomography, which, however, did not need surgical treatment. PFO in the prospective group was detected in $62 \%(95 \% \mathrm{Cl} 52-73)$ of patients. In $25 \%(95 \% \mathrm{Cl} 16-35)$, shunting was significant. A large PFO without Valsalva maneuver was detected in $12.5 \%(95 \% \mathrm{Cl} 6-21)$ of cases.

Conclusion - The use of the TEE-based protocol for the management of adult patients in a sitting position during elective neurosurgical interventions can reduce the incidence of clinically significant VAE.
\end{abstract}

Keywords: sitting position, head elevated position, venous air embolism, transesophageal echocardiography, patent foramen ovale.

Cite as Averyanov DA, Lakotko RS, Shchyogolev AV, Svistov DV, Gayvoronsky Al. The impact of transesophageal echocardiography based protocol for management of adults in the sitting position on the incidence of clinically significant venous air embolism. Russian Open Medical Journal $2020 ; 9$ : e0107.

Correspondence to Dmitry A. Averyanov. Phone: +79213863511. E-mail: dimonmed@mail.ru.

\section{Introduction}

The use of the sitting position in some neurosurgical interventions has several advantages [1, 2]. Despite that in many countries the frequency of its use has significantly decreased [3, 4]. This was facilitated by the prevailing belief that this position increases the risks of development of such complications like tension pneumocephalus, peripheral neuropathy, quadriplegia, macroglossia, and venous air embolism (VAE) [5]. VAE and paradoxical air embolism (PAE), as its most serious consequence, apparently, were the main reasons for the reduction in the frequency of the sitting position use [6]. Nevertheless, the literature data on this issue are ambiguous: some indicate the absence of additional risks [6], others indicate their increase [7]. There is even a retrospective study, which showed a greater number of intra- and postoperative complications in children who had their tumor removed in the prone rather than in the sitting position [8]. To some extent this ambiguity may be the result of differences in patient management in each particular clinic. This is evidenced by the high variability of the methods used to prevent, detect and treat possible complications [9]. At the moment, there is no widely accepted protocol regarding the combined use of these techniques.

Some steps towards developing a standardized algorithm have been taken by several authors. At the moment, at least two protocols have been proposed for management of a patient in a sitting position in order to control the risks of complications [10, 11]. The main emphasis in one of these protocols is placed on the intraoperative use of transesophageal echocardiography (TEE), as the most sensitive and specific VAE detection method [11]. The results of the protocol implementation demonstrate rather low 
incidence of overall complications, related to the sitting position and VAE in particular, which indicates that such TEE-based approach may be very promising.

The purpose of this study was to evaluate the impact of TEEbased protocol for management of adults in the sitting position during elective neurosurgical interventions on the incidence of clinically significant VAE.

\section{Material and Methods}

The presented protocol of management of patient in the sitting position was developed on the basis of Jadik S. et al. [11] work. After getting the local ethics committee approval it was implemented in the work of the neurosurgery clinic of the Military Medical Academy in 2016. Between October 2016 and March 2019, 70 patients were managed within this protocol (prospective group). In order to conduct a comparative analysis of the protocol effectiveness, retrospective data of adults scheduled for elective brain surgery during the similar preceding period (from October 2013 to March 2016) were collected (retrospective group). 85 patients were reviewed in a retrospective group. The characteristics of patients in both groups are presented in Table 1. The study design is comparative retrospective and prospective.

The protocol is a strict sequence of surgeon and anesthesiologist actions while managing a patient in the sitting position on the operating table. It focuses on the prevention, diagnosis and treatment of VAE. Within the protocol, upon admission to the operating room, the patient was provided with the following monitoring modalities - three leads electrocardiography, pulse oximetry. In order to have invasive arterial blood pressure, one of the radial arteries was cannulated under ultrasound guidance and the sensor was calibrated at the phlebostatic axis level. After bringing the patient into the sitting position, the sensor was placed at the level of the external auditory meatus and re-calibrated. The target blood pressure values during surgery were the mean arterial blood pressure of 65 $\mathrm{mmHg}$ or systolic arterial blood pressure of $100 \mathrm{mmHg}$. Norepinephrine infusion was used to manage hypotension if necessary. Prior to induction, one of the veins of the forearm was cannulated with a 16-18G catheter and, in order to reduce the chance of an intracardiac right to left gradient and hemodynamic instability development during positioning, the patient was preinfused with $0.9 \%$ sodium chloride solution $(1,000 \mathrm{ml})$ and Gelofusin $(500 \mathrm{ml})[12,13]$. The liberal tactics of infusion therapy was further used. Induction in anesthesia was provided with propofol $(1-1.5 \mathrm{mg} / \mathrm{kg})$ and fentanyl $(5-7 \mu \mathrm{g} / \mathrm{kg})$, muscle relaxation - with rocuronium bromide (0.6-1 $\mathrm{mg} / \mathrm{kg})$. Anesthesia maintenance was carried out by sevoflurane (0.9-1.1 MAK), or with propofol $(6-8 \mathrm{mg} / \mathrm{kg} / \mathrm{h})$ in case of transcranial motor evoked potentials monitoring. Fentanyl $0.1 \mathrm{mg}$ was bolused during the installation of Mayfield skull clamps and then every 30-40 minutes. After tracheal intubation, mechanical ventilation was performed in continuous mandatory ventilation mode with parameters providing a constant concentration of end-expired carbon dioxide $\left(\right.$ et $\left.\mathrm{CO}_{2}\right)$ at a level of $40 \mathrm{mmHg}$. Positive end-expirotary pressure (PEEP) was not used due to its possible negative effect on hemodynamics, low efficacy for the prevention of VAE and increased likelihood of PAE during the development of VAE [14, 15]. After induction a peripheral catheter 16-18G was inserted into one of the veins of the leg or foot to provide additional vascular access. Jugular bulb was cannulated under ultrasound guidance with a $16 \mathrm{G}$ central venous catheter in order to monitor pressure and control the efficacy of bilateral jugular compression during VAE managing. A right atrium catheter was not used due to the presence of a more sensitive VAE detection method - TEE and its obvious low efficacy in treating a clinically significant VAE when air has already entered the pulmonary bloodstream. An invasive pressure transducer was installed and calibrated at the level of the external auditory meatus [16]. The urinary bladder was catheterized to control diuresis.

Table 1. Patient characteristics

\begin{tabular}{|c|c|c|c|}
\hline Parameters & Prospective group, $n=70$ & Retrospective group, $n=85$ & Statistics \\
\hline Age, years, Me (LQ, UQ) & $45(36,58)$ & $50(19,75)$ & $\mathrm{W}=3017, \mathrm{P}=0.883$ \\
\hline Height, cm, M $\pm S D$ (min-max) & $168.5 \pm 9(150-187)$ & $169 \pm 7(152-187)$ & $W=2988, P=0.964$ \\
\hline Weight, kg, $M \pm S D$ (min-max) & $73 \pm 14(46-112)$ & $75 \pm 18(40-113)$ & $W=2747, P=0.413$ \\
\hline \multicolumn{4}{|l|}{ Sex: } \\
\hline - men, n (\%) & $33(47)$ & $34(40)$ & \multirow{2}{*}{$\begin{array}{c}\text { Chi-squared }=0.53, \\
d f=1, P=0.4651\end{array}$} \\
\hline - women, $\mathrm{n}(\%)$ & $37(53)$ & $51(60)$ & \\
\hline \multicolumn{4}{|l|}{ ASA: } \\
\hline$-1, \mathrm{n}(\%)$ & $5(7)$ & $12(14)$ & \multirow{3}{*}{$\begin{array}{c}\text { Chi-squared }=2.90, \\
d f=2, P=0.234\end{array}$} \\
\hline - II, n (\%) & $52(74)$ & $63(74)$ & \\
\hline$-\mathrm{III}, \mathrm{n}(\%)$ & $13(19)$ & $10(12)$ & \\
\hline \multicolumn{4}{|l|}{ Pathology: } \\
\hline - Cerebellopontine angle neoplasm, n (\%) & $12(17.1)$ & $15(17.6)$ & \\
\hline - Cerebellar neoplasm, n (\%) & $10(14.3)$ & $12(14.1)$ & \\
\hline - Fourth ventricle neoplasm, n (\%) & $11(15.7)$ & $13(15.3)$ & \\
\hline - Posterior cranial fossa meningioma, n (\%) & $8(11.4)$ & $10(11.8)$ & \\
\hline - Cerebellopontine angle schwannoma, n (\%) & $7(10)$ & $5(5.9)$ & \\
\hline - Occipital lobe neoplasm, n (\%) & $7(10)$ & $9(10.6)$ & \\
\hline - Neurovascular conflict, n (\%) & $6(8.6)$ & $10(11.8)$ & \\
\hline - Arnold Chiari malformation, $\mathrm{n}(\%)$ & $4(5.7)$ & $6(7.1)$ & \\
\hline - Pineal region neoplasm, n (\%) & $2(2.9)$ & $2(2.3)$ & \\
\hline - Occipital lobe AVM, n (\%) & $1(1.4)$ & $2(2.3)$ & \\
\hline - Cerebellar AVM, n (\%) & $1(1.4)$ & $1(1.2)$ & \\
\hline - Middle third of the falx meningioma, $n(\%)$ & $1(1.4)$ & $0(0)$ & \\
\hline \multicolumn{4}{|l|}{ Type of trepanning: } \\
\hline - Resectional, n (\%) & $39(56)$ & $35(41)$ & Chi-squared=2.70, \\
\hline - Osteoplastic, n (\%) & $31(44)$ & $50(59)$ & $d f=1, P=0.101$ \\
\hline
\end{tabular}

Me (LQ, UQ) - data presented as median with lower and upper quartiles; M \pm SD (min-max) - data presented as mean with standard deviation, minimum and maximum; $\mathrm{n}(\%)$ - data presented as numbers and percentages. 


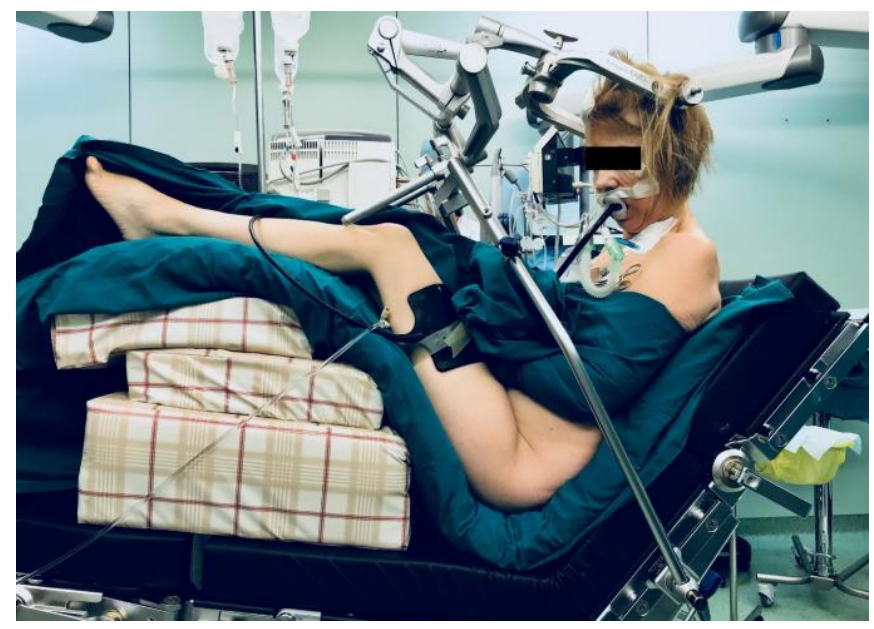

Figure 1. Patient in the sitting position.

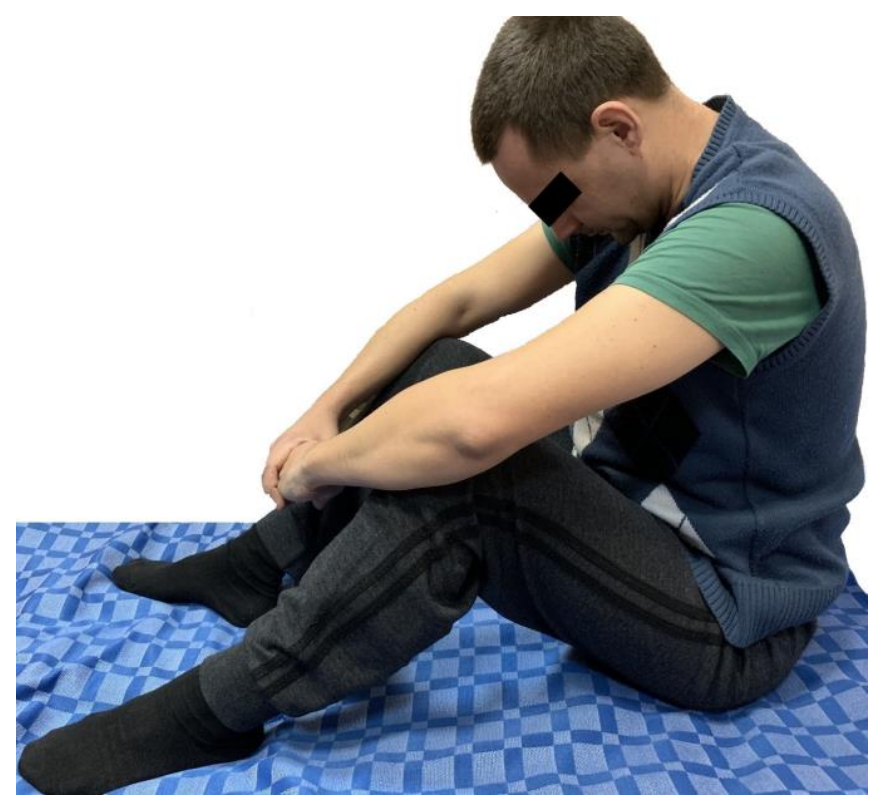

Figure 2. Positioning test in the preoperative period.

Before positioning, the esophagus of the patient was intubated with a TEE probe and a study was done to detect previously undetected severe heart pathology and right to left shunt. A Valsalva maneuver was performed to check the presence and severity of a patent foramen ovale (PFO) with the injection of an agitated $0.9 \%$ sodium chloride into the peripheral venous catheter in accordance with the technique described previously [17]. Transthoracic echocardiography in the preoperative period was not used due to its relatively low sensitivity and specificity in the detection of intracardiac right to left shunt [18]. Preoperative TEE was abandoned because of the need to carry out sedation for the procedure, which in turn decrease quality of the Valsalva maneuver [19]. The surgical team was informed of the heart study result. Subsequently, TEE (bicaval view) was used for intraoperative detection of VAE and PAE.

The patient is placed in a sitting position by steps. Initially legs are padded with thick pillows to achieve knee joints flexion about 30-45 degrees. Next, the head-holder support is attached to the back portion of the surgical table and Mayfield head-holder is secured to the patient's head. The surgical table with patient is then slowly bended anteriorly to achieve position where the hip joint is flexed to no more than 90 degrees and thoracic and cervical spine is also flexed with or without turning the head (Figure 1). Special attention is paid to the minimum space of 3-4 $\mathrm{cm}$ between the chin and sternal notch to ensure free blood flow through the internal jugular veins. Preliminary, during preoperative examination by an anesthesiologist the patient was asked to take exactly the same sitting position in a ward and questioned about complaints related to that position, such as numbness, tension, backache, etc. in order to determine the physiological limits of extremities and body flexion (Figure 2). After achieving the sitting position patient hands are laid on the abdomen. All holding constructions are then checked and locked. Body parts, that met metal platforms of the table are padded. The anesthetist aligns the pressure sensors at the level of the external auditory meatus and calibrates them. Adjusting the legs of the patient in line or above the level of the heart was considered the main component of positioning in terms of VAE prevention. The aim of leg adjustment in combination with pre-infusion was to increase preload, decrease negative effect of the sitting position on systemic hemodynamics and increase pressure in the veins and venous collectors at the surgery site [11]. Intraoperative monitoring of somatosensory and transcranial motor evoked potentials was not used because of insufficient, in our opinion, validity and feasibility of their use to detect damage to the spinal cord tracts.

Craniotomy from the moment of skin incision to the opening of the dura mater was considered as the most dangerous period of the surgery in terms of VAE development. Neck compression was performed each 5-7 minutes throughout craniotomy and during every VAE development, which was not related to intravenous administration of drugs. The effectiveness of compression was monitored by a pressure sensor in the jugular bulb. Jugular bulb pressure of more than $5 \mathrm{mmHg}$ during the compression was considered effective. At the same time, the surgeon was immediately informed. His actions included the search and closure of the air entry point in the surgical wound (irrigation with saline, bone waxing, coagulation with an electrocautery, sealing and / or suturing of a sinus defect, etc.). Hemodynamics, if necessary, was controlled using norepinephrine. The inspiratory oxygen fraction was increased to $100 \%$ in case of desaturation. With the inefficiency of the above measures, the angle of operating table inclination was decreased to reduce the heart-surgical wound pressure gradient.

The primary endpoint for evaluating the effectiveness of this protocol was considered to be a decrease in the incidence of clinically significant VAE in the prospective group in comparison with the retrospective one. In the prospective group, VAE with Tuebingen grade 3-5 was considered clinically significant [20]. In this group, the PFO incidence and severity and the effect of the number of episodes of VAE per case on its maximum severity during surgery were also analyzed. Right-to-left shunting was considered significant when more than 10 microbubbles of air appeared in the frame in the left atrium for 3-6 cardiac cycles after the APL valve was released during intravenous administration of the agitated solution within Valsalva maneuver. Any complication in the postoperative period believed to be associated with the position of the patient on the table during the surgery was recorded. 
Table 2. Summary of VAE episodes (Tuebingen VAE Grades)

\begin{tabular}{lc}
\hline VAE grade & Number of patients \\
\hline - Tuebingen I, n (\%) & $24(34.3)$ \\
- Tuebingen II, n (\%) & $6(8.6)$ \\
- Tuebingen III*, n (\%) & $4(5.7)$ \\
- Tuebingen IV*, n (\%) & $1(1.4)$ \\
- Tuebingen $\mathrm{V}^{*}, \mathrm{n}(\%)$ & 0 \\
\hline
\end{tabular}

* - clinically significant. $\mathrm{n}(\%)$ - data presented as numbers and percentages.

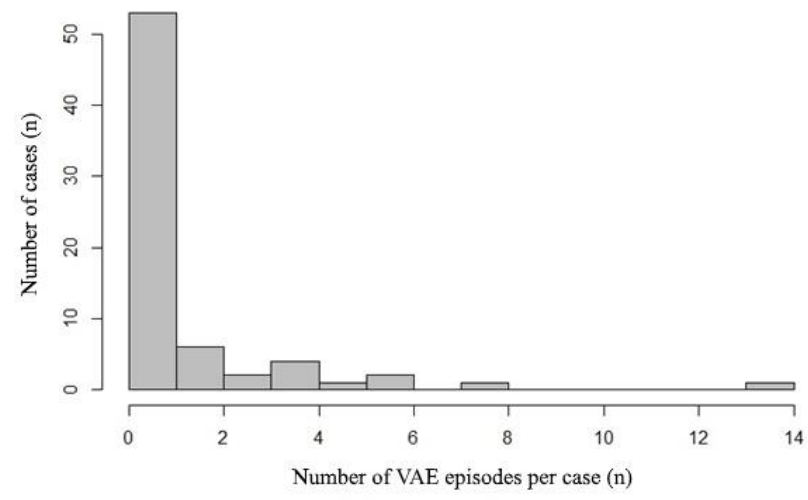

Figure 3. Number of VAE episodes per case histogram.

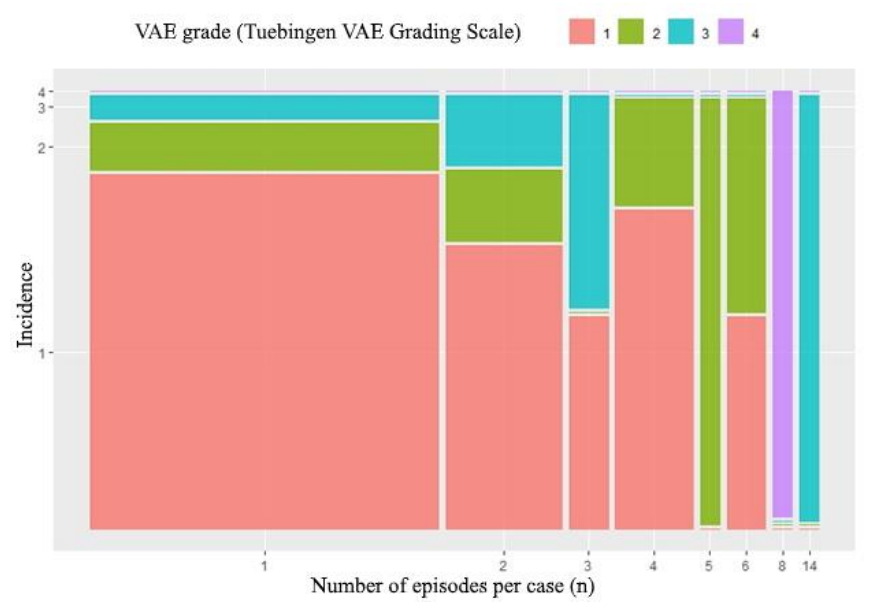

Figure 4. Incidence of the most severe VAE per case and number of VAE episodes per case histogram.

Medical records of patients in retrospective group were analyzed. Evidences of intraoperative VAE development in the anesthesia protocol was sought. The etCO2 decrease of more than $3 \mathrm{~mm} \mathrm{Hg}$ with constant minute volume lung ventilation according to the anesthesia protocol with / without changing of other gas exchange and hemodynamics indicators was considered clinically significant.

Statistical analysis of the results was made in $\mathrm{R}$ version 3.5.1 (RStudio version 1.1). Quantitative data with a normal distribution are presented as mean, standard deviation and range. Testing the hypothesis for normal distribution was carried out using graphical (histogram and quantile diagram) and criteria (Shapiro-Wilk test) methods. Quantitative data with a non-normal distribution and qualitative rank data are presented as medians and quartiles, nominal as frequencies and, in some cases, 95\% confidence intervals (exact Clopper-Pearson method). Pearson's chi-square was used to compare frequencies an expected value in any of the cells of the two by two table was less than 5 . A linear-by-linear association test with the assessment of its strength by the Somers $D$ criterion was used to identify the relationship between the frequency of VAE in the observation and its maximum severity. The severity of VAE acted as a dependent variable. The significance level at which the null hypothesis of the absence of differences in the groups was rejected was considered 0.05 .

\section{Results}

$50 \%$ (95\% $\mathrm{Cl} 38-62)$ of patients in prospective group developed VAE during surgery. Their Tuebingen grades are presented in Table 2. Clinically significant VAE, however, was only in 7.1\% (95\% $\mathrm{Cl}$ : 2.3-15.8) of observations. In 16 cases, the number of episodes was more than one (Figure 3 ). The number of episodes of VAE in the observation was reliably associated with the maximum severity of VAE during the observation $(Z=4.11, p<0.001)$. Incidence of the most severe VAE per case and number of VAE episodes per case histogram is shown in Figure 4. A moderate strength relationship was determined between them (SomersDelta $=0.43,95 \% \mathrm{Cl}: 0.17-0.7$ ). All cases of VAE were successfully terminated within the described protocol. None of the patients had to be moved to a horizontal position to terminate VAE. Not a single case of paradoxical air embolism was detected in a series of observations. None of the patients has got a neurological deficit or cardiopulmonary complications associated with the position on the surgical table in the postoperative period. Pneumocephalus was found in $100 \%$ of cases on head computed tomography, which, however, did not need surgical treatment.

PFO in the prospective group was detected in $62 \%$ (95\% Cl: $52-$ 73 ) of patients. In $25 \%$ ( $95 \% \mathrm{Cl}: 16-35)$, shunting was significant. A large PFO without Valsalva maneuver was detected in $12.5 \%$ (95\% $\mathrm{Cl}$ : 6-21) of cases.

The frequency of the clinically significant VAE in the retrospective group was $23.5 \%(95 \% \mathrm{Cl}: 15-34)$ and was $16.4 \%$ higher than the frequency in the prospective group (chisquare $=7.6197, d f=1, p=0.005$ ).

\section{Discussion}

The study was aimed at the evaluation of the impact of TEEbased protocol for management of adults in the sitting position during elective neurosurgical interventions on the incidence of clinically significant VAE. It was found that the use of a standardized approach using TEE allowed to reduce the incidence of clinically significant VAE by $16.4 \%$. This fact may indicate its positive effect on patient safety during surgery in this position.

The authors of some publications indicate that sitting positions during neurosurgical interventions lose popularity among neurosurgeons and one of the main reasons for this is the high incidence of VAE [6]. Despite that fact, leading foreign hospitals widely apply this position, which is reflected in several modern articles [21, 22]. In opposite to existing opinion, their practice demonstrates a low incidence of undesirable perioperative events. The incidence of clinically significant VAE, when TEE was used as part of the patient management protocol, is only 1.6-9.6\% [11, 20], which correlates well with the prospective data obtained in our study. The present protocol has in our opinion very little differences from the authors complexes of methods to detect and 
prevent complications associated with the position and especially with the VAE. The frequency of clinically significant VAE in descriptive studies from centers where the main method of VAE detection in adult brain surgery were precordial Doppler or etCO was more than $20 \%$. A comparison of the various protocols is presented in Table 3.

One of the probable reasons for the constant popularity of the sitting position in large neurosurgical centers is, apparently, their academic nature with a comprehensive understanding of the VAE issues and other position associated complications. The regular nature of performing such kind of surgery contributed undoubtedly to the creation of a uniform approach to the patient management in this position using the most effective methods of prevention, detection and treatment of possible complications. The most sensitive method to detect the most common complication, VAE, is currently ultrasound - precordial doppler or TEE [9]. Precordial doppler is able to detect VAE with a bolus injection of only $0.25 \mathrm{ml}$ of air, whereas the next most sensitive method, etCO2, detects only quick venous entry of $0.4 \mathrm{ml} / \mathrm{kg}$ of air, which in a patient weighing $70 \mathrm{~kg}$ is $28 \mathrm{ml}$ [23]. TEE has a slightly greater sensitivity $(0.2 \mathrm{ml}$ with a bolus injection) [24]. At least one of two specific monitoring options (precordial doppler or TEE) is used in all currently published management protocols for neurosurgery patients in sitting position $[10,11]$. High sensitivity, apparently, allows early detection of VAE, without bringing the episode of air embolism to the development of clinical signs. This is evidenced by the large difference between the overall frequency of VAE and clinically significant VAE, which according to the results of the study was $52 \%$ and $7.1 \%$, respectively. This can be also evidenced to some extent by a decrease in the incidence of clinically significant VAE by $16.4 \%$ when a standardized TEE-based approach was implemented in our clinic.

The number of VAE episodes during surgery may be more than one [20]. In the study in more than $50 \%$ of patients with VAE their number was more than one with a maximum of 14 such episodes per case. The appearance of respiratory and hemodynamic disorders in general (i.e. clinical significance) and their severity in particular (Tuebingen scale grades 3-5) obviously depends on the total air volume, which is directly affected by the frequency and duration of episodes of VAE that somehow gets into the pulmonary blood flow. This fact was partially confirmed in the study when a reliable relationship of moderate strength between the maximum severity of VAE and the number of episodes per observation was found. Until now, this relationship has not been investigated in the available literature, and therefore the obtained data can be considered new.

Regarding the various methods of ultrasound monitoring, it should be noted that despite the invasiveness and complexity, the possibilities of TEE in comparison with precordial doppler are much wider. TEE allows to detect right to left shunt, identify PAE and confirm the location of the tip of the central venous catheter in the right atrium. According to the results of this study, blood shunt through PFO was detected in $65 \%$ of patients. The literature provides data on a significantly lower proportion of PFO in the population $[25,26]$. This may be due to the use of other PFO diagnostic criteria (the number of bubbles in the frame, the number of cardiac cycles), and the use of less sensitive methods (transthoracic echocardiography, transcranial doppler, with/without contrast techniques, with / without standardized Valsalva maneuver, biopsy research) by authors.

Table 3. Techniques aimed at prevention and detection of possible sitting position complications and the incidence of clinically significant $\mathrm{VAE}$ (etCO ${ }_{2}$ decrease $>3-5 \mathrm{mmHg}$ ) in adult patients scheduled for brain surgery in the sitting position in various neurosurgical clinics

\begin{tabular}{|c|c|c|c|c|c|}
\hline Feature & $\begin{array}{l}\text { Present protocol, } \\
2019\end{array}$ & $\begin{array}{l}\text { Jadik S., } \\
2009[11]\end{array}$ & $\begin{array}{c}\text { Choque-Velasquez J., } \\
2018 \text { [21] }\end{array}$ & $\begin{array}{l}\text { Ammirati M., } \\
2013[10]\end{array}$ & $\begin{array}{l}\text { Dilmen O.K., } \\
2011[28]\end{array}$ \\
\hline PFO detection before/after induction: & $-/+$ & $+/-$ & $-/-$ & $-/+$ & $-/-$ \\
\hline$-\mathrm{TCD}$ & - & - & - & - & - \\
\hline - TTE & - & - & - & - & - \\
\hline - TEE & + & + & - & + & - \\
\hline - Valsalva maneuver & + & + & - & - & - \\
\hline Modified sitting position* & + & + & - & + & - \\
\hline $\begin{array}{l}\text { Invasive BP at the level of external auditory meatus } \\
\text { (foramen of Monro) }\end{array}$ & + & $\mathrm{n} / \mathrm{a}$ & + & $\mathrm{n} / \mathrm{a}$ & + \\
\hline Target $\mathrm{BP}(\mathrm{MAP}>60 \mathrm{mmHg}$ and/or $\mathrm{SAP}>100 \mathrm{mmHg}$ ) & + & $\mathrm{n} / \mathrm{a}$ & + & $\mathrm{n} / \mathrm{a}$ & $\mathrm{n} / \mathrm{a}$ \\
\hline CVC single-/multiorifice (Bunegin-Albin) & $-1-$ & $+/-$ & $-1-$ & $+1-$ & ++- \\
\hline JBP monitoring & + & - & - & - & - \\
\hline SSEP and TCMEP monitoring & - & + & - & - & - \\
\hline Colloid and/or crystalloid preinfusion & + & $\mathrm{n} / \mathrm{a}$ & + & $\mathrm{n} / \mathrm{a}$ & + \\
\hline Antigravity (or antishock) trousers & - & - & + & - & - \\
\hline Hypercapnia & + & - & - & + & - \\
\hline$P E E P \geq 5 \mathrm{~cm} \mathrm{H} \mathrm{H}_{2}$ & - & + & - & + & + \\
\hline \multicolumn{6}{|l|}{ VAE detection: } \\
\hline - TEE & + & + & - & - & - \\
\hline - Precordial doppler: & - & - & + & + & - \\
\hline Clinically significant VAE, \% $(95 \% \mathrm{Cl})$ & $\begin{array}{c}7.1 \\
(2.3-15.8)^{* *}\end{array}$ & $\begin{array}{c}1.6 \\
(0.3-4.6)^{* *}\end{array}$ & $\begin{array}{c}28.5 \\
(17.3-42.2)^{* * *}\end{array}$ & $\begin{array}{c}26.8 \\
(14.2-42.9)^{* * *}\end{array}$ & $\begin{array}{c}20.4 \\
(17.3-23.9)^{* * *}\end{array}$ \\
\hline
\end{tabular}

PFO - patent foramen ovale, TCD - transcranial doppler, TTE - transthoracic echocardiography, TEE - transesophageal echocardiography, BP - blood pressure, MAP - mean arterial pressure, SAP - systolic arterial pressure, CVC - central venous catheter, CVP - central venous pressure, VAE - venous air embolism, JBP - jugular bulb pressure, SSEP - somatosensory evoked potentials, TcMEP - transcranial motor evoked potentials, etCO ${ }_{2}$ - end-tidal carbon dioxide, PEEP - positive end-expiratory pressure, $\mathrm{Cl}$ - confidence interval (Clopper Pearson exact test), n/a - not available. * - Legs of the patient above heart level, $* *-V A E$ diagnostic threshold et $\mathrm{CO}_{2}>3 \mathrm{mmHg}, * * *-\mathrm{VAE}$ diagnostic threshold etCO ${ }_{2}>5 \mathrm{mmHg}$. 
Despite the high overall frequency of PFO, the danger, apparently, can only be when shunt was detected without the use of the Valsalva maneuver, which was found in $12.5 \%$ of cases. In fact, any episode of VAE in such patients will be accompanied by PAE. That will be true even in the event of completely abandoning the use of PEEP during surgery, as a factor contributing to the disruption of the normal left to right pressure gradient in the atria [14]. The final decision regarding the application of the sitting position, however, is made by the surgeon, weighing the risk and benefits of its use. In the present study, despite the presence of shunt without Valsalva maneuver in some patients, surgery was done without changing the previously selected tactics regarding the patient's position on the operating table. It should be said that such practice is not unique. The authors of some publications question the fact that it is necessary to use the PFO as an absolute contraindication to the use of the sitting position $[20,26,27]$. Nevertheless, this issue requires further study.

This study demonstrated that despite the VAE risk in sitting neurosurgery patients, the use of a standardized perioperative protocol based on TEE can reduce the incidence of clinically significant VAE.

\section{Conclusion}

The use of the TEE-based protocol for the management of adult patients in a sitting position during elective neurosurgical interventions can reduce the incidence of clinically significant VAE.

\section{Study limitations}

The limitation of this work is the conclusion on the effectiveness of the presented protocol which is based on comparison with retrospective data. That can be a subject to distortion in one direction or another for a number of known uncontrolled reasons. Unfortunately, conducting a comparative prospective study in one institution having a gold standard for intraoperative VAE detection is highly likely to be considered unethical. A multicenter study is difficult, due to the need to include a neurosurgical center where specific monitoring is not used, when the overall popularity of this position is extremely low and the approach to patient management with regard to positioning, invasive pressure monitoring, preinfusion, PEEP, etc. may vary significantly.

\section{Conflict of interest}

The authors declare that they have no conflict of interest.

\section{Ethical approval}

All procedures performed in studies involving human participants were in accordance with the ethical standards of the institutional research committee and with the 1964 Helsinki declaration and its later amendments or comparable ethical standards.

\section{References}

1. Himes BT, Mallory GW, Abcejo AS, Pasternak J, Atkinson JLD, Meyer $\mathrm{FB}$, et al. Contemporary analysis of the intraoperative and perioperative complications of neurosurgical procedures performed in the sitting position. J Neurosurg 2017; 127(1): 182-188. https://doi.org/10.3171/2016.5.JNS152328.

2. Black S, Ockert DB, Oliver WC Jr, Cucchiara RF. Outcome following posterior craniectomy in patients in the sitting or horizontal positions.
Anesthesiology 1988; 69(1): 49-56. https://doi.org/10.1097/00000542198807000-00008.

3. Kida $\mathrm{H}$, Nishikawa $\mathrm{N}$, Matsunami $\mathrm{K}$, Kawahito $\mathrm{M}$, Ota M, Miyao $\mathrm{S}$. Sitting position in the neurosurgery: the results of a questionnaire sent to neurosurgeons of medical colleges. Masui 2000; 49(5): 566-569. https://www.ncbi.nlm.nih.gov/pubmed/10846394.

4. Elton RJ, Howell RS. The sitting position in neurosurgical anaesthesia: a survey of British practice in 1991. Br J Anaesth 1994; 73(2): 247-248. https://doi.org/10.1093/bja/73.2.247.

5. Ganslandt O, Merkel A, Schmitt H, Tzabazis A, Buchfelder M, Eyupoglu I, et al. The sitting position in neurosurgery: indications, complications and results. a single institution experience of 600 cases. Acta Neurochir (Wien) 2013; 155(10): 1887-1893. https://doi.org/10.1007/s00701013-1822-x.

6. Saladino A, Lamperti M, Mangraviti A, Legnani FG, Prada FU, Casali C, et al. The semisitting position: analysis of the risks and surgical outcomes in a contemporary series of 425 adult patients undergoing cranial surgery. J Neurosurg 2017; 127(4): 867-876. https://doi.org/10.3171/2016.8.JNS16719.

7. Israelian LA, Shimanskiĭ VN, Otamanov DA, Poshataev VK, Lubnin Alu. Patient positioning on the operating table in neurosurgery: sitting or lying. Anesteziol Reanimatol 2013; (4): 18-26. Russian. https://www.ncbi.nlm.nih.gov/pubmed/24341037.

8. Orliaguet GA, Hanafi M, Meyer PG, Blanot $S$, Jarreau MM, Bresson D, et al. Is the sitting or the prone position best for surgery for posterior fossa tumours in children? Paediatr Anaesth 2001; 11(5): 541-547. https://doi.org/10.1046/j.1460-9592.2001.00733.x.

9. Gracia I, Fabregas N. Craniotomy in sitting position: anesthesiology management. Curr Opin Anaesthesiol 2014; 27(5): 474-483. https://doi.org/10.1097/ACO.00000000000000104.

10. Ammirati M, Lamki TT, Shaw AB, Forde B, Nakano I, Mani M. A streamlined protocol for the use of the semi-sitting position in neurosurgery: a report on 48 consecutive procedures. J Clin Neurosci 2013; 20(1): 32-34. https://doi.org/10.1016/j.jocn.2012.05.037.

11. Jadik S, Wissing H, Friedrich K, Beck J, Seifert V, Raabe A. A standardized protocol for the prevention of clinically relevant venous air embolism during neurosurgical interventions in the semisitting position. Neurosurgery 2009; 64(3): 533-538. https://doi.org/10.1227/01.NEU.0000338432.55235.D3.

12. Colohan AR, Perkins NA, Bedford RF, Jane JA. Intravenous fluid loading as prophylaxis for paradoxical air embolism. I Neurosurg 1985; 62(6): 839-842. https://doi.org/10.3171/jns.1985.62.6.0839.

13. Lindroos AC, Niiya $T$, Randell $T$, Romani $R$, Hernesniemi J, Niemi $T$. Sitting position for removal of pineal region lesions: the Helsinki experience. World Neurosurg 2010; 74(4-5): 505-513 https://doi.org/10.1016/j.wneu.2010.09.026.

14. Perkins NA, Bedford RF. Hemodynamic consequences of PEEP in seated neurological patients-implications for paradoxical air embolism. Anesth Analg 1984; 63(4): 429-432. https://www.ncbi.nlm.nih.gov/pubmed/6367545.

15. Toung TJ, Aizawa H, Traystman RJ. Effects of positive end-expiratory pressure ventilation on cerebral venous pressure with head elevation in dogs. J Appl Physiol (1985) 2000; 88(2): 655-661. https://doi.org/10.1152/jappl.2000.88.2.655.

16. Drummond JC, Hargens AR, Patel PM. Hydrostatic gradient is important - blood pressure should be corrected. APSF Newsletter 2009; 24(1): 6. https://www.apsf.org/article/hydrostatic-gradient-isimportant-blood-pressure-should-be-corrected.

17. Averyanov DA, Lakotko RS, Khomenko EA, Shchegolev AV. Patent Foramen Ovale Incidence and Severity in Neurosurgical Patients. Anesteziologiya i Reanimatologiya 2018; (3): 54-57. Russian. https://doi.org/10.17116/anaesthesiology201803154.

18. Pearson AC, Labovitz AJ, Tatineni S, Gomez CR. Superiority of transesophageal echocardiography in detecting cardiac source of embolism in patients with cerebral ischemia of uncertain etiology. $J$ 
Am Coll Cardiol 1991; 17(1): 66-72 https://doi.org/10.1016/07351097(91)90705-e.

19. Pinto FJ. When and how to diagnose patent foramen ovale Heart 2005; 91(4): 438-440. https://doi.org/10.1136/hrt.2004.052233.

20. Feigl GC, Decker K, Wurms M, Krischek B, Ritz R, Unertl K, et al. Neurosurgical procedures in the semisitting position: evaluation of the risk of paradoxical venous air embolism in patients with a patent foramen ovale. World Neurosurg 2014; 81(1): 159-164. https://doi.org/10.1016/j.wneu.2013.01.003.

21. Choque-Velasquez J, Colasanti R, Resendiz-Nieves JC, GonzálesEchevarría KE, Raj $R$, Jahromi $B R$, et al. Praying sitting position for pineal region surgery: an efficient variant of a classic position in neurosurgery. World Neurosurg 2018; 113: e604-e611. https://doi.org/10.1016/j.wneu.2018.02.107.

22. Gupta P, Rath GP, Prabhakar H, Bithal PK. Complications related to sitting position during pediatric neurosurgery: an institutional experience and review of literature. Neurol India 2018; 66(1): 217-222. https://doi.org/10.4103/0028-3886.222852.

23. Edmonds-Seal J, Prys-Roberts C, Adams AP. Air embolism. A comparison of various methods of detection. Anaesthesia 1971; 26(2): 202-208. https://doi.org/10.1111/j.1365-2044.1971.tb04763.x.

24. Furuya $H$, Suzuki $T$, Okumura $F$, Kishi $Y$, Uefuji $T$. Detection of air embolism by transesophageal echocardiography. Anesthesiology 1983; 58(2): 124-129. https://doi.org/10.1097/00000542-198302000-00004.

25. Hagen PT, Scholz DG, Edwards WD. Incidence and size of patent foramen ovale during the first 10 decades of life: an autopsy study of 965 normal hearts. Mayo Clin Proc 1984; 59(1): 17-20. https://doi.org/10.1016/s0025-6196(12)60336-x.

26. Engelhardt M, Folkers W, Brenke C, Scholz M, Harders A, Fidorra H, et al. Neurosurgical operations with the patient in sitting position: analysis of risk factors using transcranial Doppler sonography. $\mathrm{Br} \mathrm{J}$ Anaesth 2006; 96(4): 467-472. https://doi.org/10.1093/bja/ael015.

27. Klein J, Juratli TA, Weise M, Schackert G. A systematic review of the semi-sitting position in neurosurgical patients with patent foramen ovale: how frequent is paradoxical embolism? World Neurosurg 2018; 115: 196-200. https://doi.org/10.1016/j.wneu.2018.04.114.

28. Dilmen OK, Akcil EF, Tureci E, Tunali $Y$, Bahar M, Tanriverdi T, et al. Neurosurgery in the sitting position: retrospective analysis of 692 adult and pediatric cases. Turk Neurosurg 2011; 21(4): 634-640 https://doi.org/10.5137/1019-5149.JTN\%20.4974-11.0.

Authors:

Dmitry A. Averyanov - MD, PhD, Lecturer, Department of Anesthesiology and Resuscitation, S.M. Kirov Military Medical Academy, Saint-Petersburg, Russia. https://orcid.org/0000-0003-4353-4953.

Roman S. Lakotko - MD, PhD student, Department of Anesthesiology and Resuscitation, S.M. Kirov Military Medical Academy, Saint-Petersburg, Russia. https://orcid.org/0000-0003-0941-013X.

Aleksey V. Shchyogolev - MD, DSc, Professor, Head of the Department of Anesthesiology and Resuscitation, S.M. Kirov Military Medical Academy, Saint-Petersburg, Russia. https://orcid.org/0000-0001-6431-439X.

Dmitry V. Svistov - MD, DSc, Associate Professor, Head of the Department of Neurosurgery, S.M. Kirov Military Medical Academy, Saint-Petersburg, Russia. https://orcid.org/0000-0002-3922-9887.

Aleksey I. Gayvoronsky - MD, DSc, Associate Professor, Senior Lecturer, Department of Neurosurgery, S.M. Kirov Military Medical Academy, SaintPetersburg, Russia. https://orcid.org/0000-0003-1886-5486. 